\title{
Mitochondria in Cancer Energy Metabolism: Culprits or Bystanders?
}

\author{
Aekyong Kim \\ School of Pharmacy, Catholic University of Daegu, Gyeongbuk, Korea
}

(Received November 18, 2015; Revised December 15, 2015; Accepted December 17, 2015)

\begin{abstract}
Cancer is a disease characterized by uncontrolled growth. Metabolic demands to sustain rapid proliferation must be compelling since aerobic glycolysis is the first as well as the most commonly shared characteristic of cancer. During the last decade, the significance of metabolic reprogramming of cancer has been at the center of attention. Nonetheless, despite all the knowledge gained on cancer biology, the field is not able to reach agreement on the issue of mitochondria: Are damaged mitochondria the cause for aerobic glycolysis in cancer? Warburg proposed the damaged mitochondria theory over 80 years ago; the field has been testing the theory equally long. In this review, we will discuss alterations in metabolic fluxes of cancer cells, and provide an opinion on the damaged mitochondria theory.
\end{abstract}

Key words: Cancer, Mitochondria, Metabolism, Aerobic glycolysis, Glutaminolysis, Anaplerosis, Cataplerosis

\section{INTRODUCTION}

As our knowledge on cancer expands, the firm belief that cancer is a genetic disease is seriously challenged (1). A rather provocative proposal is rising in the field of oncology: Cancer is a metabolic disease. Mitochondrial respiratory dysfunction is openly proposed as the origin of cancer (2).

To avoid unseen impediments under clinical settings, we have intensively investigated to gain solid insight into how cancer arises and what could be the genetic and epigenetic alterations responsible for cancer. Throughout multistage oncogenesis, cells with the best fitness for survival and proliferation will be selected. Genetic and epigenetic alterations endowing cells for evolutionary fitness will be rapidly disseminated among descendants. As tumor microenvironments change over the course of cancer progression, these alterations will vary considerably, reflecting a temporal as well as spatial complexity of oncogenesis.

With increasing appreciation of the importance of cancer energy metabolism during oncogenesis, in this review we

\footnotetext{
Correspondence to: Aekyong Kim, School of Pharmacy, Catholic University of Daegu, Gyeongbuk 712-702, Korea

E-mail: aekyongkim@cu.ac.kr
}

This is an Open-Access article distributed under the terms of the Creative Commons Attribution Non-Commercial License (http:// creativecommons.org/licenses/by-nc/3.0) which permits unrestricted non-commercial use, distribution, and reproduction in any medium, provided the original work is properly cited. will scrutinize recent scientific evidence to properly define the role of mitochondria in cancer energy metabolism. For this purpose, detailed underlying molecular mechanisms utilized by cancer cells during metabolic reprogramming are beyond the scope of this review. Hence, we will limit our discussion to a couple of examples by p53 tumor suppressor (TP53) and proto-oncogene c-Myc, and even then only partially. This review aims to present how cellular energy metabolism is manipulated by cancer cells in relation to catabolism and anabolism (Fig. 1), and clarify the exact nature of mitochondrial involvement in oncogenesis.

Metabolic reprogramming occurs in cancer cells. Otto Warburg, the Nobel laureate, reported in the 1920s that cancer cells had elevated glycolysis even under aerobic conditions (3); the phenomenon is often called the Warburg effect. Dysfunctional mitochondria have long been the prime suspect for the Warburg effect (4). Over six decades ago, Weinhouse, another distinguished biochemist, also suggested that characteristics of energy metabolism in cancer cells could been defined as high glycolytic flux in the cytosol (5). However, according to Weinhouse, mitochondrial respiration in cancer cells is functional (6) but suppressed as a consequence of heightened glycolytic flux in the cytosol (5). Inhibition of mitochondrial respiration by addition of glucose to cells is called the reversed Pasteur effect or, more often, the Crabtree effect. From the viewpoint of the Crabtree effect, mitochondria are not guilty of causing accelerated glycolysis, but are innocent bystanders (7). 


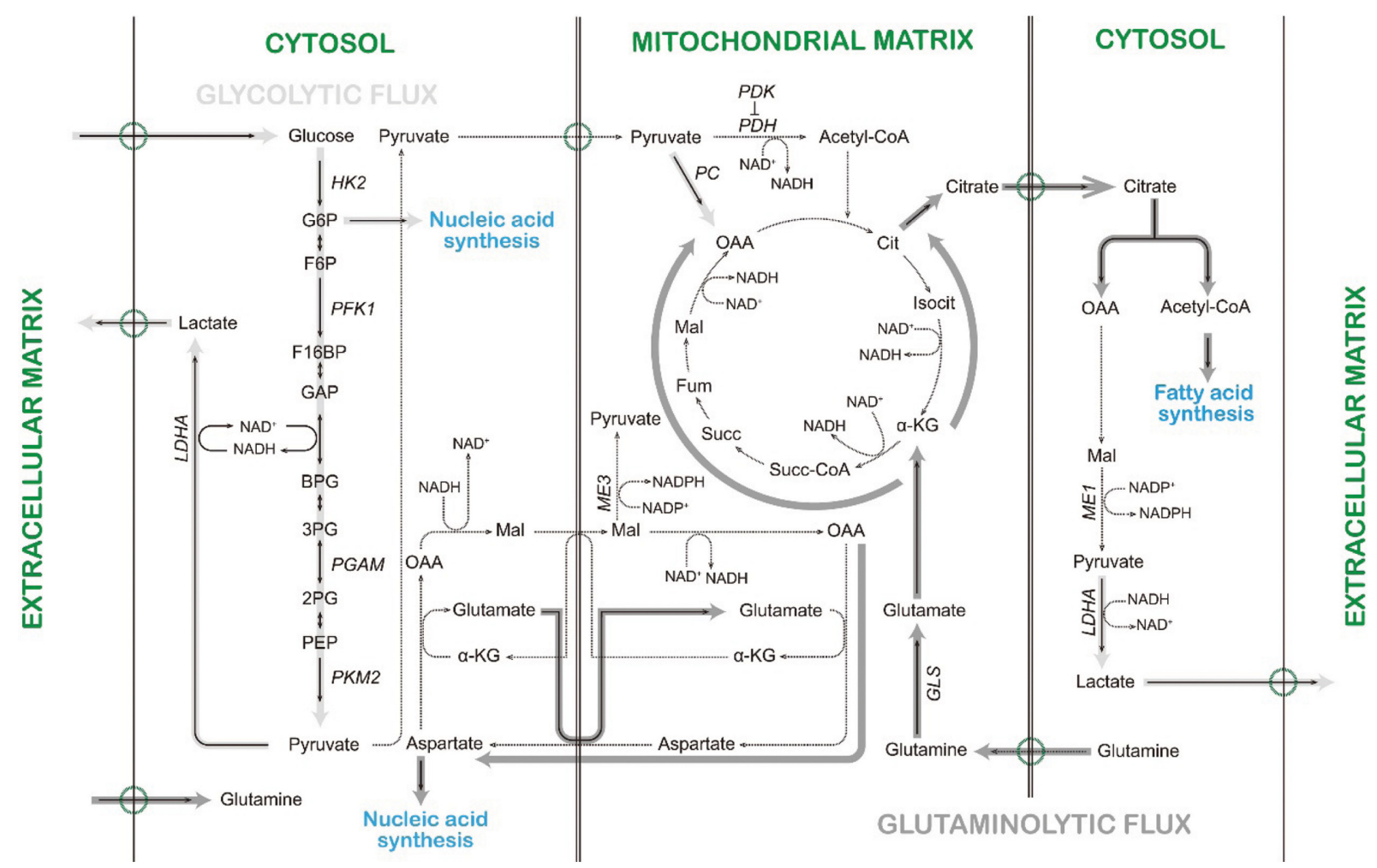

Fig. 1. Altered metabolic fluxes in cancer cells. Abbreviation: G6P, glucose-6-phosphate; F6P, fructose-6-phosphate; F16BP, fructose-1,6bisphosphate; GAP, glyceraldehyde 3-phosphate; BPG, 1,3-bisphosphoglycerate; 3PG, 3-phosphoglycerate; 2PG, 2-phosphoglycerate; PEP, phosphoenolpyruvate; Cit, citrate; Isocit, isocitrate; $\alpha-K G$, $\alpha$-ketoglutarate; Succ-CoA, succinyl-CoA; Succ, succinate; Fum, fumarate; Mal, malate; OAA, oxaloacetate; HK2, hexokinase 2; PFK1, phosphofructokinase 1; PGAM, phosphoglycerate mutase; PKM2, pyruvate kinase M2; LDHA, lactate dehydrogenase A; PDH, pyruvate dehydrogenase; PDK, PDH kinase; PC, pyruvate carboxylase; ME1 and 3, malic enzyme 1 and 3; GLS, glutaminase.

Over the past five decades, the field of cancer biology seems to have been unable to reach a definitive conclusion on the issue of mitochondria in cancer energy metabolism: culprit or innocent bystander. On the other hand, the seemingly paradoxical preference of cancer cells for glycolysis has stood strong. Underlying molecular mechanisms by which cancer cells enhance aerobic glycolysis have been unraveled and major reprogramming of cellular energy metabolism is now appreciated as one of the hallmarks of cancer (8).

Glycolytic flux is increased in cancer cells. As Warburg observed, cancer cells often take up more glucose into the cytoplasm compared to non-malignant cells. TP53 decreases expression of glucose transporters at the transcriptional level (9); tumor-associated mutant p53 promotes the translocation of glucose transporter 1 to the cytoplasmic membrane (10). The conversion of glucose to glucose6-phosphate is mediated by hexokinases (HK). c-Myc induces HK2 at the transcriptional level $(11,12)$.

Although both cellular glucose uptake and metabolic activation of glucose to glucose-6-phosphate could result in enhanced glycolytic flux, the ultimate commitment of glucose to glycolysis is made during the conversion of fructose-6-phosphate to fructose-1,6-bisphosphate by phosphofructokinase 1 (PFK1). A critical factor controlling this step is the concentration of fructose-2,6-bisphosphate (F26BP). TP53 suppresses glycolysis by transcriptionally upregulating TP53-induced glycolysis and apoptosis regulator (TIGAR) that enzymatically degrades F26BP (13). Phosphoglycerate mutase (PGAM) converts 3-phosphoglycerate to 2-phosphoglycerate. TP53 transcriptionally upregulates a ubiquitin ligase by which PGAM 1 is polyubiquitinated and targeted for proteasomal degradation (14).

The last step of glycolysis, catalyzed by pyruvate kinase (PK), converts phosphoenolpyruvate (PEP) to pyruvate. Among 12 known isoforms, PKM2 is strongly associated with tumorigenicity (15). The isoform switch of PKM1 to PKM2 occurs post-transcriptionally through microRNAs (16-18). In addition, recent studies suggest that PKM2 is upregulated due to DNA hypomethylation on the PKM gene (19) and by c-Myc at the transcriptional level $(12,20)$.

If present in excess, pyruvate is enzymatically converted to lactate by lactate dehydrogenase (LDH). Lactate is then 
exported out of the cell via a proton-linked monocarboxylate transporter (MCT) (21). c-Myc transcriptionally upregulates LDHA (12) and MCT1 (22) that participate in a tumor-specific lactate shuttle (21).

Pyruvate metabolism is decoupled from glycolytic flux in cancer cells. Mitochondria serve as hubs where cellular carbon is traded; the Krebs cycle ensures impartial trades of cellular carbon according to physiological demands. Some carbons are completely oxidized to $\mathrm{CO}_{2}$, providing electrons for ATP production, whereas some must return to the cytosol in different packages for anabolic reactions. In cancer, the Krebs cycle seems to favor carbon usage for anabolic reactions.

Pyruvate enters the mitochondrial matrix through a mitochondrial pyruvate carrier (MPC) $(23,24)$. MPCs are underexpressed in many cancers and seem to be involved in the stemness of cancer cells (25). Since molecular identification of MPC genes was only recently accomplished $(23,24)$, genetic regulation of MPCs, especially by tumor suppressors and oncogenes, will be revealed soon.

To enter the Krebs cycle, pyruvate is oxidatively decarboxylated to acetyl-CoA by pyruvate dehydrogenase (PDH), the activity of which is inhibited by pyruvate dehydrogenase kinase (PDK). Down-regulation of PDH (26) and upregulation of PDK (27) are correlated with poor prognosis of cancer patients. c-Myc upregulates PDK1 at the transcriptional level (11).

Pyruvate also enters the Krebs cycle as oxaloacetate which condenses with acetyl-CoA to form citrate, which is the first step in the Krebs cycle. On the other hand, pyruvate carboxylase (PC) catalyzes the conversion of pyruvate to oxaloacetate, consuming one ATP during the reaction. It is only recently that increased expression and activity of PC have been shown to serve as the means of major anaplerotic input in cancer cells $(28,29)$. Although the promoter of the PC gene has been analyzed (30), tumor-specific regulation of the PC gene has yet to be investigated at the transcriptional level.

Glutaminolytic and glycolytic fluxes are coupled in cancer cells. Cancer cells use glutamate as another important carbon source for anaplerosis. Glutamine enters cells via a sodium-dependent neutral amino acid transporter, alanineserine-cysteine transporter 2 (ASCT2). While carriers in the cytoplasmic membrane for glutamine uptake have been extensively studied, the exact molecular nature of the mitochondrial glutamine carrier still remains elusive (31). Once in the mitochondrial matrix, deamination of glutamine is mediated by glutaminase (GLS). Expression of ASCT2 and GLS increases during multistep carcinogenesis (32). While ASCT2 is a direct transcriptional target of n-Myc in neuroblastoma cells (33), c-Myc increases GLS expression by transcriptional suppression of microRNAs (34).
Carbons from glutamate enter the Krebs cycle as $\alpha$-ketoglutarate $(\alpha-K G)$. Through stepwise oxidation in the Krebs cycle, glutamate can be completely oxidized to $\mathrm{CO}_{2}$ providing hydrogen/electrons for mitochondrial oxidative phosphorylation (OXPHOS). Instead, carbons from glutamate can leave the Krebs cycle as malate and be transported out to the cytosol via pyruvate/malate shuttle. Oxidative decarboxylation of malate to pyruvate can be catalyzed by malic enzyme 1 (ME1) producing NADPH. Thomson and colleagues proposed that cancer cells would preferentially employ the glutaminolytic pathway to supply cytosolic NADPH for anabolic reactions (35). As in aerobic glycolysis of cancer cells, pyruvate is then reduced to lactate and transported out of the cell. Hence, similarly to aerobic glycolysis of cancer cells, glutaminolysis can be defined as an energy-producing pathway in which glutamine is oxidized via parts of the Krebs cycle and then partially oxidized carbons are removed from the cell as lactate.

Glutaminolytic flux is increased in some cancers (31). Moreover, experimental data suggest that cancer cells utilize glutamate not for energy production but for anabolic reaction. Intermediates in the Krebs cycle can serve as building blocks for macromolecules necessary for growth and proliferation. According to a recent study (36), in proliferating cells, the physiological significance of glutaminolysis is to provide nitrogen for de novo synthesis of nucleobases. Glutamine and aspartate provide nitrogen to pyrimidine and purine bases of nucleotides. When metabolic pathways in tumor spheroids were simultaneously analyzed by intracellular metabolite profiles (37), glutaminolysis was active but cytosolic ME1 was not. These points suggest that glutamate enters the Krebs cycle as $\alpha-K G$, is sequentially oxidized to oxaloacetate and then leaves the mitochondria, after amination, as aspartate via the malate/ aspartate shuttle. Indeed, a study reports that cancer cells actively synthesize several amino acids including aspartate (37).

In contrast, carbons from glutaminolysis leave the Krebs cycle as citrate and are transported out to the cytosol via citrate carriers. In the cytosol, citrate may serve as a carbon source for de novo synthesis of fatty acids. In fact, glutamine contributes as much as glucose to the production of lipogenic acetyl-CoA in brown adipocytes (38). In 1984, Parlo and Coleman reported that hepatomas accumulate excess amounts of cholesterol in the mitochondrial membranes via de novo cholesterogenesis using lipogenic acetylCoA originating from mitochondrial citrate (39).

Under specific experimental conditions such as hypoxia $(40,41)$ or impaired mitochondrial OXPHOS (42), glutamate can be converted to citrate for de novo fatty acid synthesis. $\mathrm{NADP}^{+}$-dependent isocitrate dehydrogenases (IDHs), for example cytosolic IDH1 $(40,42)$ and mitochondrial IDH2 (41), catalyze $\mathrm{CO}_{2}$-dependent reductive carboxylation of $\alpha-K G$ to isocitrate. Then, aconitase converts isocitrate to 
citrate for lipogenesis. Hence, glutaminolysis by reductive carboxylation operates through the backward pathway of the truncated Krebs cycle $(7,43)$.

PFK1 fine-tunes metabolic fluxes in cancer cells. Cancer cells demand more ATP, NADPH and intermediates from the Krebs cycle for anabolic reactions to ensure their uncontrollable proliferation (7). Hence, catabolic degradation of glucose and glutamine increase to meet their demands for macromolecules. Furthermore, cellular metabolic fluxes must be modulated to meet their demands for de novo synthesis of nucleotides, proteins and lipids. Cancer cells may simply use tumor suppressor genes and oncogenes as metabolic switches to modulate metabolic fluxes to their best advantage (44).

As we look more closely into how cancer cells rewire metabolic fluxes, it seems that cancer cells strategically mutilate allosteric inhibition of the reaction catalyzed by PFK1. First, the loss of TP53 in cancer cells results in the absence of TIGAR expression. Consequently, the level of F26BP remains elevated, and irreversible commitment to glycolysis will be sustained (13). Second, lactate inhibits PFK1 (45). Hence, glycolytic tumors often upregulate MCT4 and actively remove lactate from the cytosol to prevent cytosolic $\mathrm{pH}$ change (21).

PEP is another allosteric inhibitor for PFK1 (46). PKMs convert PEP to pyruvate. Cancer cells preferentially upregulate PKM2, the kinase activity of which is lower than PKM1 (47) or even absent (48). Considering the strong correlation between aerobic glycolysis and oncogenesis, it seems counterintuitive that cancer cells prefer PKM2 over PKM1 (49). Nonetheless, it is widely accepted that the bottleneck at the metabolic intersection for pyruvate rewires cellular catabolic pathways favorable for de novo synthesis of macromolecules $(7,49,50)$. Furthermore, when the jam at the metabolic intersection of pyruvate is removed by experimental means, the Warburg effect seems relieved (7). From such a point of view, mitochondria in cancer cells are considered as neither irreversibly damaged nor guilty of causing cancer.

The inhibitory effects of PEP on aerobic glycolysis remain in cancer cells with PKM2 expression. An alternative reaction by which PEP can be dephosphorylated to pyruvate is proposed. PEP is shown to phosphorylate the catalytic histidine $^{11}$ of PGAM1 by direct phosphate transfer (46). His ${ }^{11}$ phosphorylated PGAM1 becomes activated and rewires glycolytic flux towards de novo synthesis of NADPH, nucleotides and amino acids (51). PGAM1 is now considered as a fine metabolic tuner coordinating glycolytic flux and anabolic pathways for optimal metabolic fluxes for proliferation (52).

Citrate and ATP are additional allosteric inhibitors for PFK1 (53). The increased lipogenic demand of cancer cells is met by mitochondrial cataplerotic citrate condensed from glucose-derived acetyl-CoA and glutamate-derived oxaloacetate $(35,53)$. Therefore, it is unlikely that citrate would be accumulated in cancer cells to a high enough level to allosterically inhibit PFK1. Indeed, the level of citrate is lower in tumors than in normal tissues (54).

On the other hand, allosteric inhibition of PFK1 by ATP could impose a rather serious problem in maintaining glycolytic flux. Produced in excess, ATP will inhibit glycolytic flux. Warburg calculated that cancer cells would produce $10 \%$ more ATP than normal cells (4). According to a recent tumor metabolomics study (54), nucleotide content and energy charge were similar in normal and tumor tissues.

Hence, it may be advantageous for cancer cells to maintain a low local ATP concentration. While the reaction of PKM is linked to ATP production, phosphorylation of PGAM1 by PEP generates pyruvate without ATP production (46). Therefore, PKM2-independent dephosphorylation of PEP to pyruvate seems to be an adequate underlying molecular regulation mechanism that cancer cells employ to ensure enhanced glycolytic flux while rewiring metabolic fluxes.

\section{The Krebs cycle is decoupled from OXPHOS in cancer}

cells. As we have postulated above, if the allosteric inhibition of PFK1 by ATP poses serious limitation, then allosteric inhibition of the Krebs cycle by ATP and NADH (55) must be challenging obstacles in maintaining enhanced metabolic fluxes for anabolism in cancer cells. To maintain a low local ATP concentration, it seems valid to minimize ATP production via mitochondrial respiration. One may describe such a phenomenon as decoupling the Krebs cycle from mitochondrial OXPHOS. Contrary to the prevailing hypothesis that insufficient ATP production due to impaired mitochondrial respiration is compensated through elevated aerobic glycolysis, cancer cells may intentionally shut off mitochondrial OXPHOS to maximize metabolic fluxes for increased demands for anabolic reactions.

Findings from a recent study (56) could be interpreted from the aspect of decoupling of the Krebs cycle and OXPHOS. Ectonucleoside triphosphate diphosphohydrolase 5 (ENTPD5) is a uridine diphosphatase located in the endoplasmic reticulum. During N-glycosylation reactions, ENTPD5 hydrolyzes UDP to UMP and Pi. Cytosolic ATP is rapidly hydrolyzed to AMP to replenish the UTP pool for regeneration of UDP-glucose. With increased ENTPD5 expression, lactate production increased, but mitochondrial respiration did not change (56). To properly execute N-glycosylation reactions, glycolytic flux is increased and rewired to support nucleotide and hexosamine synthesis (57). Hence, an increase in glycolytic flux is expected, yet failure to increase mitochondrial respiration is not.

These phenomena were interpreted as demonstrating that cancer cells would not change metabolic flux to supplement ATP $(56,57)$. Nonetheless, it is still not explained why 
cells block mitochondria from supplying ATP via OXPHOS. Unlike studies in which glutaminolysis by reductive carboxylation contributed to fatty acid synthesis under hypoxia $(40,41)$ or in respiration-defective mitochondria $(42)$, the study on ENTPD5-mediated aerobic glycolysis (56) cannot relate the failure to increase mitochondrial respiration to defects in the mitochondrial respiration system in the experimental model of choice. In a recent study, it has been suggested that suboptimal ATP levels could rewire metabolic fluxes (58). Therefore, this study may suggest that cancer cells intentionally keep ATP at suboptimal levels in order to sustain high metabolic fluxes.

ETC complexes are decoupled from ATP production in cancer cells. In mitochondrial OXPHOS, electrons from electron donors $\mathrm{NADH}$ and $\mathrm{FADH}_{2}$ flow through electron transfer chain (ETC) complexes to the ultimate electron acceptor molecular oxygen. Electron transfer is directly coupled to proton translocation, which creates a protonmotive force across the mitochondrial inner membrane. Electron-motive force from electron donors is indirectly coupled to phosphoryl transfer potential via proton-motive force. Finally, a high-energy phosphoanhydride bond is formed in ATP, harnessing free energy released from electron donors (59). The magnitude of proton-motive force inversely regulates the electron transfer rate (60). The oxygen consumption rate usually reflects the electron transfer rate in ETC complexes. Therefore, cellular energy demand is accurately reflected in mitochondrial membrane potential and the oxygen consumption rate.

In general, mitochondrial respiration implies reduction of oxygen molecules to water, whereas ATP production refers to phosphoryl bond formation in ADP to ATP. Therefore, the former involves ETC complexes, and the latter is limited by ATP synthase. In consideration of recent studies $(46,56,61,62)$, it seems increasingly feasible that cancer cells decouple not only the Krebs cycle from mitochondrial OXPHOS but mitochondrial respiration from ATP production. Two independent studies suggested that ETC complexes in mitochondrial OXPHOS are essential to ensure rapid proliferation $(61,62)$. Studies demonstrate that in de novo synthesis of pyrimidine nucleotides, aspartate is supplied by cataplerotic reactions. ETC complexes oxidize $\mathrm{NADH}$ that is generated during cataplerotic aspartate synthesis, therefore maintaining the $\mathrm{NAD}^{+} / \mathrm{NADH}$ ratio $(61,62)$. Since the function of ETC complexes is successfully replaced by electron acceptors, the function of ATP production in mitochondrial OXPHOS seems dispensable (61). In these studies, ETC complexes are decoupled from ATP production, and coupled instead to the Krebs cycle.

In conclusion, recent studies demonstrate that cancer cells rewire metabolic fluxes to support enhanced anabolic reaction. Some examples are the following: cancer cells couple glycolytic and glutaminolytic fluxes, decouple pyruvate metabolism from glycolytic flux, and decouple ATP production from ETC complexes, but couple the Krebs cycle with ETC complexes. Hence, it seems that cancer cells have growth advantage over normal cells not because they synthesize absolutely more macromolecules but because they strategically operate metabolic fluxes under limiting circumstances to supply proper amounts of macromolecules at an appropriate time.

\section{CLOSING REMARKS}

During the early and middle parts of the $20^{\text {th }}$ century, cellular energy metabolism was the research topic for many distinguished biochemists. Warburg was one of them; he was awarded the Nobel Prize for his work on mitochondrial respiratory enzymes. Hans Krebs published his paper on the Krebs cycle in 1937 (63); he was also awarded the Nobel Prize, in 1953.

Until the 1970s, mitochondria stayed at the center of numerous biochemists' research interest. After 20 years' long silence, mitochondria made a strong comeback through their role in apoptosis (64). It has been only a preview! They seem to be coming back to the mainstage with a big splash; this time it will likely be through their role in metabolic reprogramming of cancer. As we are entering into a new era for mitochondria, we must discard a rather fixed view on mitochondria as the cellular powerhouse. When scientists looked at them as biochemists, mitochondrial OXPHOS and bioenergetics were discovered. Only when we started exploring them as biologists, was their hidden role in apoptosis unraveled. To properly understand their role in cancer energy metabolism, we must investigate them as physiologists and explore mitochondrial physiology. With recent advances in technology (64), we now can provide physiological meaning to mitochondrial biology.

Considering what has been revealed on mitochondrial functional biology and mitochondrial physiology, Warburg's theory on aerobic glycolysis is mostly correct, except that mitochondria are not damaged, but simply dysfunctional. On the other hand, he was wrong on the 'irreversible' part, since mitochondrial function is modulated, not permanently changed, to accommodate metabolic demands from the host.

Moreover, Weinhouse's claim is correct in that mitochondrial respiration is operational in cancer cells. However, what he meant by mitochondrial respiration is limited to the bioenergetics of mitochondria, whereas the physiological meaning of mitochondrial respiration includes, at the least, modulation of metabolic fluxes. Therefore, he was also wrong because mitochondrial respiration is bioenergetically operational but physiologically malfunctional, and therefore dysfunctional.

Indeed, mitochondria are not innocent bystanders during metabolic reprogramming of cancer cells. It is yet impetu- 
ous to reach a verdict on the charge of instigating metabolic alteration in cancer cells. Nonetheless, one thing is already obvious. Without full cooperation/assistance from mitochondria, cancer cells cannot succeed in evolutionary competition during oncogenesis by means of genetic and epigenetic alterations alone. Therefore, we must carefully investigate how mitochondria accommodate requests from the host (alterations in mitochondrial physiology), and devise tools to weaken their collaboration to outsmart them during oncogenesis.

\section{ACKNOWLEDGEMENTS}

This study was supported by grants from the National Research Foundation of Korea funded by the Korean government (no. 2012-0006753 \& no. 2012-0008391) to Aekyong Kim.

\section{REFERENCES}

1. Seyfried, T.N., Flores, R.E., Poff, A.M. and D'Agostino, D.P. (2014) Cancer as a metabolic disease: implications for novel therapeutics. Carcinogenesis, 35, 515-527.

2. Coller, H.A. (2014) Is cancer a metabolic disease? Am. J. Pathol., 184, 4-17.

3. Warburg, O., Wind, F. and Neglers, E. (1930) On the metabolism of tumors in the body in In metabolism of tumors Ed. Arnold Constable and Co. Press, London, pp. 254-270.

4. Koppenol, W.H., Bounds, P.L. and Dang, C.V. (2011) Otto Warburg's contributions to current concepts of cancer metabolism. Nat. Rev. Cancer, 11, 325-337.

5. Crabtree, H.G. (1929) Observations on the carbohydrate metabolism of tumours. Biochem. J., 23, 536-545.

6. Weinhouse, S. (1956) On respiratory impairment in cancer cells. Science, 124, 267-269.

7. Senyilmaz, D. and Teleman, A.A. (2015) Chicken or the egg: Warburg effect and mitochondrial dysfunction. F1000Prime Rep., 7, 41 .

8. Hanahan, D. and Weinberg, R.A. (2011) Hallmarks of cancer: the next generation. Cell, 144, 646-674.

9. Schwartzenberg-Bar-Yoseph, F., Armoni, M. and Karnieli, E. (2004) The tumor suppressor p53 down-regulates glucose transporters GLUT1 and GLUT4 gene expression. Cancer Res., 64, 2627-2633.

10. Zhang, C., Liu, J., Liang, Y., Wu, R., Zhao, Y., Hong, X., Lin, M., Yu, H., Liu, L., Levine, A.J., Hu, W. and Feng, Z. (2013) Tumour-associated mutant p53 drives the Warburg effect. Nat. Commun., 4, 2935.

11. Kim, J.W., Gao, P., Liu, Y.C., Semenza, G.L. and Dang, C.V. (2007) Hypoxia-inducible factor 1 and dysregulated c-Myc cooperatively induce vascular endothelial growth factor and metabolic switches hexokinase 2 and pyruvate dehydrogenase kinase 1. Mol. Cell Biol., 27, 7381-7393.

12. Kim, J.W., Zeller, K.I., Wang, Y., Jegga, A.G., Aronow, B.J., O'Donnell, K.A. and Dang, C.V. (2004) Evaluation of myc Ebox phylogenetic footprints in glycolytic genes by chromatin immunoprecipitation assays. Mol. Cell Biol., 24, 5923-5936.
13. Bensaad, K., Tsuruta, A., Selak, M.A., Vidal, M.N., Nakano, K., Bartrons, R., Gottlieb, E. and Vousden, K.H. (2006) TIGAR, a p53-inducible regulator of glycolysis and apoptosis. Cell, 126, 107-120.

14. Mikawa, T., Maruyama, T., Okamoto, K., Nakagama, H., Lleonart, M.E., Tsusaka, T., Hori, K., Murakami, I., Izumi, T., Takaori-Kondo, A., Yokode, M., Peters, G., Beach, D. and Kondoh, H. (2014) Senescence-inducing stress promotes proteolysis of phosphoglycerate mutase via ubiquitin ligase Mdm2. J. Cell Biol., 204, 729-745.

15. Christofk, H.R., Vander Heiden, M.G., Harris, M.H., Ramanathan, A., Gerszten, R.E., Wei, R., Fleming, M.D., Schreiber, S.L. and Cantley, L.C. (2008) The M2 splice isoform of pyruvate kinase is important for cancer metabolism and tumour growth. Nature, 452, 230-233.

16. Zhan, C., Yan, L., Wang, L., Ma, J., Jiang, W., Zhang, Y., Shi, Y. and Wang, Q. (2015) Isoform switch of pyruvate kinase M1 indeed occurs but not to pyruvate kinase M2 in human tumorigenesis. PLoS One, 10, e 0118663.

17. Taniguchi, K., Ito, Y., Sugito, N., Kumazaki, M., Shinohara, H., Yamada, N., Nakagawa, Y., Sugiyama, T., Futamura, M., Otsuki, Y., Yoshida, K., Uchiyama, K. and Akao, Y. (2015) Organ-specific PTB1-associated microRNAs determine expression of pyruvate kinase isoforms. Sci. Rep., 5, 8647.

18. David, C.J., Chen, M., Assanah, M., Canoll, P. and Manley, J.L. (2010) HnRNP proteins controlled by c-Myc deregulate pyruvate kinase mRNA splicing in cancer. Nature, 463, 364368.

19. Desai, S., Ding, M., Wang, B., Lu, Z., Zhao, Q., Shaw, K., Yung, W.K., Weinstein, J.N., Tan, M. and Yao, J. (2014) Tissue-specific isoform switch and DNA hypomethylation of the pyruvate kinase PKM gene in human cancers. Oncotarget, 5, $8202-8210$.

20. Xu, X., Li, J., Sun, X., Guo, Y., Chu, D., Wei, L., Li, X., Yang, G., Liu, X., Yao, L., Zhang, J. and Shen, L. (2015) Tumor suppressor NDRG2 inhibits glycolysis and glutaminolysis in colorectal cancer cells by repressing c-Myc expression. Oncotarget, 6, 26161-26176.

21. Draoui, N. and Feron, O. (2011) Lactate shuttles at a glance: from physiological paradigms to anti-cancer treatments. Dis. Models Mech., 4, 727-732.

22. Doherty, J.R., Yang, C., Scott, K.E., Cameron, M.D., Fallahi, M., Li, W., Hall, M.A., Amelio, A.L., Mishra, J.K., Li, F., Tortosa, M., Genau, H.M., Rounbehler, R.J., Lu, Y., Dang, C.V., Kumar, K.G., Butler, A.A., Bannister, T.D., Hooper, A.T., Unsal-Kacmaz, K., Roush, W.R. and Cleveland, J.L. (2014) Blocking lactate export by inhibiting the Myc target MCT1 Disables glycolysis and glutathione synthesis. Cancer Res., 74, 908-920.

23. Herzig, S., Raemy, E., Montessuit, S., Veuthey, J.L., Zamboni, N., Westermann, B., Kunji, E.R. and Martinou, J.C. (2012) Identification and functional expression of the mitochondrial pyruvate carrier. Science, 337, 93-96.

24. Bricker, D.K., Taylor, E.B., Schell, J.C., Orsak, T., Boutron, A., Chen, Y.C., Cox, J.E., Cardon, C.M., Van Vranken, J.G., Dephoure, N., Redin, C., Boudina, S., Gygi, S.P., Brivet, M., Thummel, C.S. and Rutter, J. (2012) A mitochondrial pyruvate carrier required for pyruvate uptake in yeast, Drosophila, and humans. Science, 337, 96-100. 
25. Schell, J.C., Olson, K.A., Jiang, L., Hawkins, A.J., Van Vranken, J.G., Xie, J., Egnatchik, R.A., Earl, E.G., DeBerardinis, R.J. and Rutter, J. (2014) A role for the mitochondrial pyruvate carrier as a repressor of the Warburg effect and colon cancer cell growth. Mol. Cell, 56, 400-413.

26. Sun, X.R., Sun, Z., Zhu, Z., Guan, H.X., Li, C.Y., Zhang, J.Y., Zhang, Y.N., Zhou, H., Zhang, H.J., Xu, H.M. and Sun, M.J. (2015) Expression of pyruvate dehydrogenase is an independent prognostic marker in gastric cancer. World J. Gastroenterol., 21, 5336-5344.

27. Hur, H., Xuan, Y., Kim, Y.B., Lee, G., Shim, W., Yun, J., Ham, I.H. and Han, S.U. (2013) Expression of pyruvate dehydrogenase kinase- 1 in gastric cancer as a potential therapeutic target. Int. J. Oncol., 42, 44-54.

28. Sellers, K., Fox, M.P., Bousamra, M. 2nd, Slone, S.P., Higashi, R.M., Miller, D.M., Wang, Y., Yan, J., Yuneva, M.O., Deshpande, R., Lane, A.N. and Fan, T.W. (2015) Pyruvate carboxylase is critical for non-small-cell lung cancer proliferation. J. Clin. Invest., 125, 687-698.

29. Cardaci, S., Zheng, L., MacKay, G., van den Broek, N.J., MacKenzie, E.D., Nixon, C., Stevenson, D., Tumanov, S., Bulusu, V., Kamphorst, J.J., Vazquez, A., Fleming, S., Schiavi, F., Kalna, G., Blyth, K., Strathdee, D. and Gottlieb, E. (2015) Pyruvate carboxylation enables growth of SDH-deficient cells by supporting aspartate biosynthesis. Nat. Cell Biol., 17, 1317-1326.

30. Wutthisathapornchai, A., Vongpipatana, T., Muangsawat, S., Boonsaen, T., MacDonald, M.J. and Jitrapakdee, S. (2014) Multiple E-boxes in the distal promoter of the rat pyruvate carboxylase gene function as a glucose-responsive element. PLoS One, 9, e102730.

31. Matés, J.M., Segura, J.A., Campos-Sandoval, J.A., Lobo, C., Alonso, L., Alonso, F.J. and Márquez, J. (2009) Glutamine homeostasis and mitochondrial dynamics. Int. J. Biochem. Cell Biol., 41, 2051-2061.

32. Cetindis, M., Biegner, T., Munz, A., Teriete, P., Reinert, S. and Grimm, M. (2015) Glutaminolysis and carcinogenesis of oral squamous cell carcinoma. Eur. Arch. Otorhinolaryngol., 1-9. doi:10.1007/s00405-015-3543-7.

33. Ren, P., Yue, M., Xiao, D., Xiu, R., Gan, L., Liu, H. and Qing, G. (2015) ATF4 and N-Myc coordinate glutamine metabolism in MYCN-amplified neuroblastoma cells through ASCT2 activation. J. Pathol., 235, 90-100.

34. Gao, P., Tchernyshyov, I., Chang, T.C., Lee, Y.S., Kita, K., Ochi, T., Zeller, K.I., De Marzo, A.M., Van Eyk, J.E., Mendell, J.T. and Dang, C.V. (2009) c-Myc suppression of miR$23 \mathrm{a} / \mathrm{b}$ enhances mitochondrial glutaminase expression and glutamine metabolism. Nature, 458, 762-765.

35. DeBerardinis, R.J., Mancuso, A., Daikhin, E., Nissim, I., Yudkoff, M., Wehrli, S. and Thompson, C.B. (2007) Beyond aerobic glycolysis: transformed cells can engage in glutamine metabolism that exceeds the requirement for protein and nucleotide synthesis. Proc. Natl. Acad. Sci. U.S.A., 104, 19345-19350.

36. Meng, M., Chen, S., Lao, T., Liang, D. and Sang, N. (2010) Nitrogen anabolism underlies the importance of glutaminolysis in proliferating cells. Cell Cycle, 9, 3921-3932.

37. Hunnewell, M.G. and Forbes, N.S. (2010) Active and inactive metabolic pathways in tumor spheroids: determination by GC-
MS. Biotechnol. Prog., 26, 789-796.

38. Yoo, H., Stephanopoulos, G. and Kelleher, J.K. (2004) Quantifying carbon sources for de novo lipogenesis in wild-type and IRS-1 knockout brown adipocytes. J. Lipid Res., 45, 13241332.

39. Parlo, R.A. and Coleman, P.S. (1984) Enhanced rate of citrate export from cholesterol-rich hepatoma mitochondria. The truncated Krebs cycle and other metabolic ramifications of mitochondrial membrane cholesterol. J. Biol. Chem., 259, 9997-10003.

40. Metallo, C.M., Gameiro, P.A., Bell, E.L., Mattaini, K.R., Yang, J., Hiller, K., Jewell, C.M., Johnson, Z.R., Irvine, D.J., Guarente, L., Kelleher, J.K., Vander Heiden, M.G., Iliopoulos, O. and Stephanopoulos, G. (2011) Reductive glutamine metabolism by IDH1 mediates lipogenesis under hypoxia. Nature, 481, 380-384.

41. Wise, D.R., Ward, P.S., Shay, J.E., Cross, J.R., Gruber, J.J., Sachdeva, U.M., Platt, J.M., DeMatteo, R.G., Simon, M.C. and Thompson, C.B. (2011) Hypoxia promotes isocitrate dehydrogenase-dependent carboxylation of alpha-ketoglutarate to citrate to support cell growth and viability. Proc. Natl. Acad. Sci. U.S.A., 108, 19611-19616.

42. Mullen, A.R., Wheaton, W.W., Jin, E.S., Chen, P.H., Sullivan, L.B., Cheng, T., Yang, Y., Linehan, W.M., Chandel, N.S. and DeBerardinis, R.J. (2011) Reductive carboxylation supports growth in tumour cells with defective mitochondria. Nature, 481, 385-388.

43. Rehberg, M., Rath, A., Ritter, J.B., Genzel, Y. and Reichl, U. (2014) Changes in intracellular metabolite pools during growth of adherent MDCK cells in two different media. Appl. Microbiol. Biotechnol., 98, 385-397.

44. Levine, A.J. and Puzio-Kuter, A.M. (2010) The control of the metabolic switch in cancers by oncogenes and tumor suppressor genes. Science, 330, 1340-1344.

45. Leite, T.C., Coelho, R.G., Da Silva, D., Coelho, W.S., Marinho-Carvalho, M.M. and Sola-Penna, M. (2011) Lactate downregulates the glycolytic enzymes hexokinase and phosphofructokinase in diverse tissues from mice. FEBS Lett., 585, 92-98.

46. Vander Heiden, M.G., Locasale, J.W., Swanson, K.D., Sharfi, H., Heffron, G.J., Amador-Noguez, D., Christofk, H.R., Wagner, G., Rabinowitz, J.D., Asara, J.M. and Cantley, L.C. (2010) Evidence for an alternative glycolytic pathway in rapidly proliferating cells. Science, 329, 1492-1499.

47. Yang, W. and Lu, Z. (2015) Pyruvate kinase M2 at a glance. J. Cell Sci., 128, 1655-1660.

48. Hosios, A.M., Fiske, B.P., Gui, D.Y. and Vander Heiden, M.G. (2015) Lack of evidence for PKM2 protein kinase activity. Mol. Cell, 59, 850-857.

49. Cairns, R.A., Harris, I.S. and Mak, T.W. (2011) Regulation of cancer cell metabolism. Nat. Rev. Cancer, 11, 85-95.

50. Vander Heiden, M.G., Cantley, L.C. and Thompson, C.B. (2009) Understanding the Warburg effect: the metabolic requirements of cell proliferation. Science, 324, 1029-1033.

51. Hitosugi, T., Zhou, L., Elf, S., Fan, J., Kang, H.B., Seo, J.H., Shan, C., Dai, Q., Zhang, L., Xie, J., Gu, T.L., Jin, P., Alečkovic, M., LeRoy, G., Kang, Y., Sudderth, J.A., DeBerardinis, R.J., Luan, C.H., Chen, G.Z., Muller, S., Shin, D.M., Owonikoko, T.K., Lonial, S., Arellano, M.L., Khoury, H.J., 
Khuri, F.R., Lee, B.H., Ye, K., Boggon, T.J., Kang, S., He, C. and Chen, J. (2012) Phosphoglycerate mutase 1 coordinates glycolysis and biosynthesis to promote tumor growth. Cancer Cell, 22, 585-600.

52. Jiang, X., Sun, Q., Li, H., Li, K. and Ren, X. (2014) The role of phosphoglycerate mutase 1 in tumor aerobic glycolysis and its potential therapeutic implications. Int. J. Cancer, 135, 1991-1996.

53. Stine, Z.E. and Dang, C.V. (2013) Stress eating and tuning out: cancer cells re-wire metabolism to counter stress. Crit. Rev. Biochem. Mol. Biol., 48, 609-619.

54. Hirayama, A., Kami, K., Sugimoto, M., Sugawara, M., Toki, N., Onozuka, H., Kinoshita, T., Saito, N., Ochiai, A., Tomita, M., Esumi, H. and Soga, T. (2009) Quantitative metabolome profiling of colon and stomach cancer microenvironment by capillary electrophoresis time-of-flight mass spectrometry. Cancer Res., 69, 4918-4925.

55. Berg, J., Tymoczko, J. and Stryer, L. (2002) Entry to the Citric Acid Cycle and Metabolism Through It Are Controlled in Biochemistry Ed. New York.

56. Fang, M., Shen, Z., Huang, S., Zhao, L., Chen, S., Mak, T.W. and Wang, X. (2010) The ER UDPase ENTPD5 promotes protein N-glycosylation, the Warburg effect, and proliferation in the PTEN pathway. Cell, 143, 711-724.
57. Shirato, K., Nakajima, K., Korekane, H., Takamatsu, S., Gao, C., Angata, T., Ohtsubo, K. and Taniguchi, N. (2011) Hypoxic regulation of glycosylation via the $\mathrm{N}$-acetylglucosamine cycle. J. Clin. Biochem. Nutr., 48, 20-25.

58. Israelsen, W.J. and Vander Heiden, M.G. (2010) ATP consumption promotes cancer metabolism. Cell, 143, 669-671.

59. Berg, J., Tymoczko, J. and Stryer, L. (2002) Oxidative Phosphorylation in Biochemistry Ed. New York.

60. Bagkos, G., Koufopoulos, K. and Piperi, C. (2015) Mitochondrial emitted electromagnetic signals mediate retrograde signaling. Med. Hypotheses, 85, 810-818.

61. Sullivan, L.B., Gui, D.Y., Hosios, A.M., Bush, L.N., Freinkman, E. and Vander Heiden, M.G. (2015) Supporting aspartate biosynthesis is an essential function of respiration in proliferating cells. Cell, 162, 552-563.

62. Birsoy, K., Wang, T., Chen, W.W., Freinkman, E., AbuRemaileh, M. and Sabatini, D.M. (2015) An essential role of the mitochondrial electron transport chain in cell proliferation is to enable aspartate synthesis. Cell, 162, 540-551.

63. Krebs, H.A. and Johnson, W.A. (1937) Metabolism of ketonic acids in animal tissues. Biochem. J., 31, 645-660.

64. Pagliarini, D.J. and Rutter, J. (2013) Hallmarks of a new era in mitochondrial biochemistry. Genes Dev., 27, 2615-2627. 